\title{
Cardiac Medial Modeling and Time-Course Heart Wall Thickness Analysis
}

\author{
Hui Sun ${ }^{1}$, Brian B. Avants ${ }^{1}$, Alejandro F. Frangi ${ }^{2}$, Federico Sukno ${ }^{2}$, \\ James C. Gee ${ }^{1}$, and Paul A. Yushkevich ${ }^{1}$ \\ ${ }^{1}$ Departments of Radiology, University of Pennsylvania, Philadelphia, PA, USA \\ ${ }^{2}$ Information and Communication Technologies Department, University Pompeu \\ Fabra, Barcelona, Spain and Networking Center on Biomedical Research - \\ CIBER-BBN, Barcelona, Spain
}

\begin{abstract}
The medial model is a powerful shape representation method that models a $3 \mathrm{D}$ object by explicitly defining its skeleton (medial axis) and deriving the boundary geometry according to medial geometry. It has been recently extended to model complex shapes with multi-figures, i.e., shapes whose skeletons can not be described by a single sheet in 3D. This paper applied the medial model to a 2-chamber heart data set consisting of 428 cardiac shapes from 90 subjects. The results show that the medial model can capture the heart shape accurately. To demonstrate the usage of the medial model, the changes of the heart wall thickness over time are analyzed. We calculated the mean heart wall thickness map of 90 subjects for different phases of the cardiac cycle, as well as the mean thickness change between phases.
\end{abstract}

\section{Introduction}

With the rapid development of cardiac imaging techniques, there has been increased interest in geometric models that allow the accurate extraction of meaningful shape features and provide correspondence support for population studies. The heart walls are thin, sheet-like structures which can be effectively described by skeletons (medial axes). Medial models [12], which describe a structure by explicitly defining its skeleton and deriving the boundary geometry from the medial axis, have the ability to represent shape compactly and provide global shape features. Some of those shape features, like wall thickness and surface curvature, have the potential to provide useful information about functions of the heart [3. However, the applications of medial models on cardiac shapes are still quite limited, probably due to the complex shape of the heart [4. Here the term "complex shape" refers to the structure whose skeleton can not be described by a single curve (2D) or single sheet (3D).

One relevant work to this paper is the "centersurface method" proposed by Bolson and Sheehan [5]. It constructs a triangulated centersurface (medial axis) midway between the input endocardium and epicardium surfaces via skeletonization, and then it defines the local thickness at the vertex of the centersurface as the length of the line segment which is perpendicular to the centersurface at this 
point and lies between the endocardium and epicardium surfaces. However, the method only deals with a single heart chamber. And unlike medial models, the shape features (thickness maps) generated from different objects are not aligned, posing problems for further population comparison.

There have been efforts to model complex shapes using medial models. The difficulty of constructing medial model for complex shapes arises from the fact that the geometric relationship between the medial axis and the boundary imposes equality constraints that must be satisfied along the seam curves (curves where different medial sheets meet). Han et al proposed a "multi-figure" medial model [6] that represents each part of a complex object medially, but does not model the connections between parts in terms of medial geometry. Rather, it uses surface blending to attach a "child" single-figure medial model to its "parent". This type of model is very useful when complex objects have a "parent-child" organization of parts (like the hand, with a palm and five fingers) and the medial geometry and shape features along the connections between parts are less interesting, but it is not clear how it can be applied to structures like heart. Terriberry 7] proposed using Catmull-Clark subdivision surfaces to model the skeleton and enforced the equality constraints by locally modifying the skeleton at seam curves to use interpolating splines. This solution is elegant, but it is somewhat limited by the requirement of Catmull-Clark subdivision surfaces with quadrilateral elements being used. To the best of our knowledge, Terriberry's complex shape medial model has not yet been applied to large-scale anatomical modeling.

We have recently proposed a medial model for complex shapes which uses soft penalties to enforce the equality constraints required by medial geometry [4. It turns out to be robust, easy to implement and sufficient in practice. In this paper, as a demonstration of the medial model, we apply it to a large scale cardiac data set with 428 heart instances for a time-course wall thickness analysis.

\section{Method and Experimental Results}

\section{$2.1 \quad$ Materials}

The data set we used contains 428 heart shapes from 90 subjects consisting of both healthy subjects and patients suffering from common cardiovascular pathologies, including myocardium infarction (25), hypertrophy (21), LV dilation (6), LV aneurysm (2), RV dilation (2), LA dilation(5), RA dilation (2), and pericarditis(4). The MR images were generously provided to us by CETIR Sant Jordi Centre (Barcelona, Spain). The acquisition parameters are: TR: 3.75 4ms, TE: 1.5-1.58 ms, FA: 45, slice thickness: $8 \mathrm{C} 10 \mathrm{~mm}$, slice size: $256 \times 256$ pixels, resolution: $1.56 \times 1.56 \mathrm{~mm}$ and FOV: $400 \times 300 \mathrm{~mm}^{2}$, on a General Electric CVI 1.5 T MR facility. Expert segmentations were manually drawn on the endocardial left ventricle and right ventricle borders, and on the epicardial border of the whole heart to construct a 2-chamber heart model usually including 8-12 slices from the base to the apex. Five different phases of the cardiac cycle were segmented: End Diastole (ED),Mid Systole (MS), End Systole (ES),Diastole 1 (D1) and Diastole 2 (D2). 


\subsection{Medial Modeling of Cardiac Shapes}

The medial model approach leverages the idea of inverse skeletonization: the skeleton (medial axis) of an object is defined first and the object boundary is constructed analytically from the skeleton. The medial model for a particular object is derived by fitting a deformable medial template to the binary image mask of the object. The skeleton is described as a combination of medial manifolds $\mathbf{m}$ in $\mathcal{R}^{3}$ and a positive valued radial thickness field $R$ in $\mathcal{R}^{+}$, where the $\mathbf{m}$ and $R$ can be the user-specified mesh/surface/function. The Loop subdivision surface is used in this work to represent the medial manifolds. The equations used to derive object boundary $\mathbf{b}^{ \pm}$from the skeleton are the following

$$
\begin{gathered}
\mathbf{b}^{ \pm}=\mathbf{m}+R \mathbf{U}^{ \pm} \\
\mathbf{U}^{ \pm}=-\nabla_{m} R \pm \sqrt{1-\left\|\nabla_{m} R\right\|^{2}} \mathbf{N}_{m},
\end{gathered}
$$

where $\mathbf{N}_{m}$ is the unit normal vector of the medial manifold at point $\mathbf{m}, \mathbf{U}^{ \pm}$are unit length vectors orthogonal to $\partial O$ at $\mathbf{b}^{ \pm}$, and $\nabla_{m}$ is the Riemannian gradient of $R$ on the medial manifold.

The key difficulty of the medial model approach lies on the well-posedness of the inverse skeletonization problem; that is, given arbitrary connected surface patches $\mathbf{m}$ and an arbitrary positive field $R$, the $\{\mathbf{m}, R\}$ pair may not form the skeleton of any subject. Rather, inverse skeletonization is only possible for those $\{\mathbf{m}, R\}$ pairs which satisfy a set of equality and inequality constraints required by the medial geometry [17/4. In our algorithm, the equality and inequality constraints are both incorporated as soft penalty terms in the deformable medial model, and the equality constraints are then further enforced by a brute-force local adjustment 4 .

The need for equality constraints can be motivated by geometric facts. According to Eq. 1. one medial surface will generate two boundary surface patches. Therefore if all the boundary patches are to meet together seamlessly to form the boundary of an object, equality constraints are needed along the edges and seam curves of medial manifolds.

Along the medial edge (boundary of the medial manifolds), the following corresponding equality constraint is needed to ensure that the two boundary patches meet each other $\left(\mathbf{b}^{+}=\mathbf{b}^{-}\right)$:

$$
\left\|\nabla_{m} R\right\|-1=0 .
$$

Accordingly, a soft penalty term for violating this constraint can be put as

$$
\left(\left\|\nabla_{m} R\right\|-1\right)^{2} .
$$

Points on the medial seam curve belong to three medial manifolds $\mathbf{m}^{i}\{i=$ $1,2,3\}$ 8. Assuming they are oriented so that $\mathbf{U}^{i,+}=\mathbf{U}^{i \oplus 1,-}(\oplus$ denotes additional modulo 3 ) at the seam, then the equality constraints are

$$
\nabla_{m}^{i \oplus 2} R-\nabla_{m}^{i \oplus 1} R=\sqrt{1-\left\|\nabla_{m}^{i} R\right\|^{2}} \mathbf{N}_{m}^{i} .
$$


With some algebra, the equality constraints above can be written as

$$
\frac{\partial R}{\partial \mathbf{s}_{i}}+\sqrt{1-\left(\frac{\partial R}{\partial \mathbf{t}}\right)^{2}} \mathbf{N}_{m}^{i \oplus 1} \cdot \mathbf{N}_{m}^{i \oplus 2}=0,
$$

where $\mathbf{t}$ is the tangent vector of the seam curve, and $\mathbf{s}_{i}=\mathbf{t} \times \mathbf{N}_{m}^{i}$, which is tangent to the medial manifold $\mathbf{m}^{i}$ and orthogonal to the seam curve. Accordingly, the soft penalty for violating this constraint can be put as

$$
\left(\frac{\partial R}{\partial \mathbf{s}_{i}}+\sqrt{1-\left(\frac{\partial R}{\partial \mathbf{t}}\right)^{2}} \mathbf{N}_{m}^{i \oplus 1} \cdot \mathbf{N}_{m}^{i \oplus 2}\right)^{2} .
$$

At the seam-edge intersection, $\left\|\nabla_{m}^{i} R\right\|$ equals 1 for the medial manifold whose edge is crossing the intersection, and the other two manifolds will have angle $\pi$ (actually they merge into one manifold at the intersection point).

There are several more inequality constraints that the $\{\mathbf{m}, R\}$ pair need to satisfy, including $R>0,\left\|\nabla_{m} R\right\| \leq 1$, and the Jacobian constraint. Refer to [1] for a more detailed description.

Template-Building for Initialization. The deformable medial model fits an initial cardiac medial template to the binary segmentation of cardiac shapes; thus, prior to the fitting, a cardiac medial template needs to be generated. In theory the template can be any simple hand-created model as long as the topology configuration is correct. However, a data-driven model which is closer to the true solution obviously would help the deformable model avoid local minimums in the fitting process. In our work, the template is derived through several steps. First, a volumetric template is iteratively generated from the dataset itself using a method similar to 9 . We use the symmetric diffeomorphic registration algorithm developed by Avants et al. [10] in the volumetric template construction. In this step, the deformation fields which warp the volumetric template to individual cardiac shapes are also generated and saved. Then, the volumetric template is binarized and the Voronoi Skeleton of the binary image is computed using qhull (www.qhull.org). The Voronoi Skeleton can be further pruned if it has a lot of fuzzy branches. In our case, however, it turns out there are not a lot of fuzzy branches for the Voronoi skeleton of the template, probably because the volumetric template, which is an unbiased average of all the cardiac images, tends to have a smooth and regular shape. In this step, we do not require the Voronoi Skeleton to be pruned until it has the correct topology. The Voronoi Skeleton, however, is represented by a very dense mesh with thousands of triangles, which is not suitable for a medial template. In order to generate a quality triangulation for the skeleton mesh, vertices and triangles are placed under manual control to produce the final medial template, which has 195 vertices and 381 triangles. To initialize the deformable model for a particular cardiac shape, we warp the medial template according to the deformation field we saved in the volumetric template construction step, and we use the deformed medial template as the initialization. The pipeline is illustrated in Fig [1. 


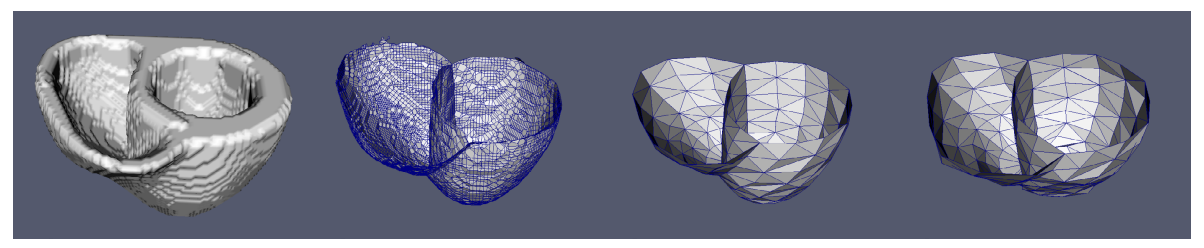

Fig. 1. Medial template building and deformable model initialization pipeline. First: the boundary of the binaried volumetric template constructed by iterative unbiased averaging algorithm. Second: the pruned Voronoi Skeleton; note that it still has some small branches and the mesh is dense. Third: the medial template constructed under manual control. Forth: example of a deformed medial template as the initialization for one cardiac shape.

Model Fitting. Similar to the method we used in 4, in order to fit a medial model to a binary segmentation of a cardiac shape, we deform the medial model defined by $\{\mathbf{m}, R\}$ to minimize an objective function which incorporates the volumetric overlap error between the interior of the deformable model and target cardiac shape. The object function also incorporates a set of prior terms, which include both penalty terms of the inequality and equality constraints required by inverse skeletonization and some regularization terms. Different from [4, where there is only one regularization term to control the quality of the medial mesh by penalizing big and small angles in the triangles, in this paper, we added another term to enforce correspondence of the medial surfaces by penalizing the distortion of medial mesh triangular area element with respect to the manually created medial template.

The model is fitted to the 428 manual segmentations of the 2-chamber heart model in a multi-resolution fashion. The initial template mesh is subdivided once in the first stage and twice in the second stage to represent the medial axis, and the target is smoothed by Gaussian kernel with variance 2 in the first stage and variance 0.6 in the second stage. The results of the fitting are illustrated in Fig 2. All the cardiac shapes are fitted with Dice overlap coefficient higher than 0.8 with an average of 0.92 . Given that the fitted cardiac shapes are from both healthy subjects and patients suffering common cardiovascular pathologies, this fitting experiment really demonstrates the robustness of our method.

\subsection{Time-Course Analysis of Heart Wall Thickness Maps}

This section is a demonstration of the usage of the cardiac medial model by analyzing the time-course changes of the heart wall thickness. Two important features of the medial model are highlighted by this experiment: First is the model's ability to derive important shape features; and second is that the representation of the skeleton in the model allows us to perform surface based statistical analysis with ease.

In order to get some idea of the cardiac shapes and thickness patterns in different phases of a cardiac cycle, we computed the mean medial manifolds 


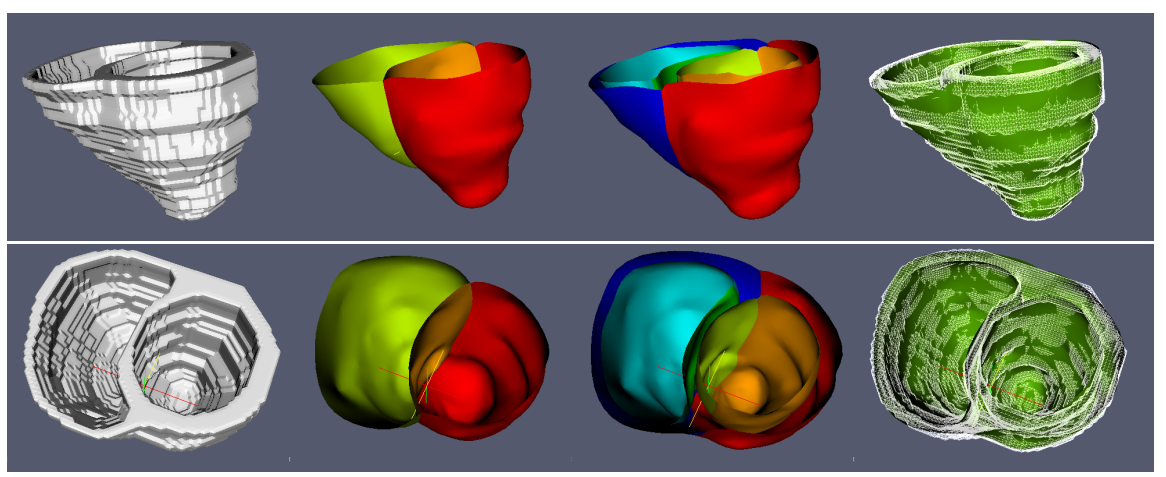

Fig. 2. An example of fitting the 2-chamber heart shape. The top row and bottom row are shown from different viewpoints. The first column is the binary segmentation of the left and right ventricles. The second column is the fitted medial model, using different color to identify medial manifolds. The third column is the model boundary: each medial manifold generates two pieces of boundary, and those boundary patches connect seamlessly. The fourth column overlays the model boundary, which is shown as green surfaces, on the binary segmentation, which is shown as white wireframe.

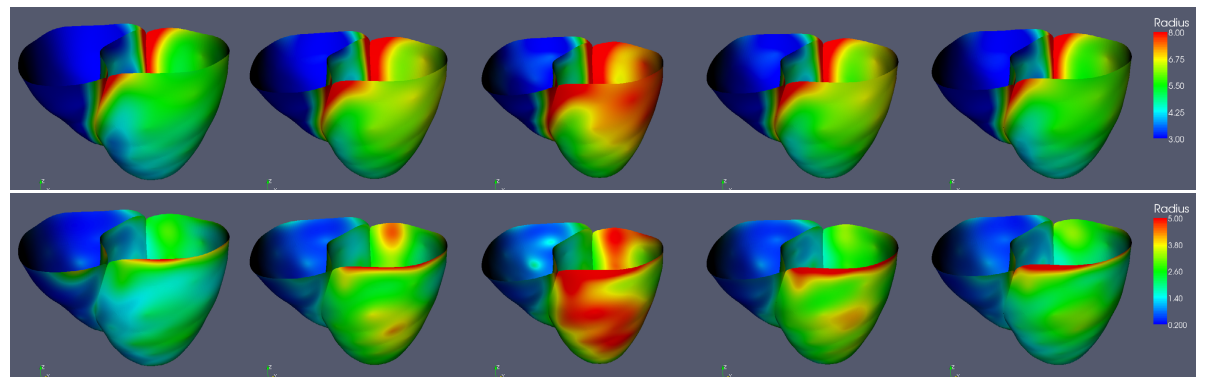

Fig. 3. The mean and variance thickness maps rendered on the mean medial shapes for five different phases of the cardiac cycle. On the top is the mean thickness map, on the bottom is the variance thickness map. From left to right, the five phases are: ED (End Diastole), MS (Mid Systole), ES (End Systole),D1 (Diastole 1) and D2 (Diastole 2).

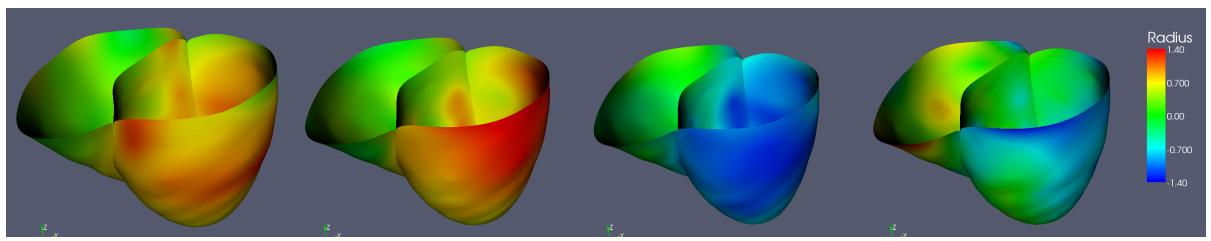

Fig. 4. The mean thickness change map between successive phases of the cardiac cycle. From left to right, the four mean change maps are: from ED to MS, from MS to ES, from ES to D1, from D1 to D2. 
and thickness map in each phase. This is possible because the medial models for those cardiac shapes are all deformed from the same template, and thus the correspondence is known. The computation of a mean model is therefore nothing but a point-wise averaging. The mean thickness maps as well as the variance thickness maps in all phases are shown in Fig 3

However, the change of the thickness map over time is still not obvious by looking at the mean thickness maps in different time. Therefore we also obtained the mean thickness change map between successive phases, which further illustrated the motion pattern over time. The mean thickness change map are shown in Fig 4. As can be seen from the figure, the thickness of the left ventricle are changing more rapidly than that of the right ventricle. The left ventricle heart wall becomes thicker from ED (End Diastole) to MS (Mid Systole) to ES (End Systole), and then becomes thinner from ES to D1 (Diastole 1) to D2 (Diastole 2 ). Also, the left ventricle wall changes more during the time from ES to D1 than during the time from D1 to D2.

\section{Discussion}

The skeleton is a natural representation for the thin, sheet-like heart wall since it summarizes the cardiac shape effectively. In this paper, the capability of medial model to represent the two-chamber cardiac shape is demonstrated by a experiment on large scale data set consisting of both healthy subjects and patients. The results show that the model can capture the heart shape accurately. The paper also demonstrated the usage of medial model by a time-course heart wall thickness analysis experiment. However, the focus this experiment is the demonstration of the ability of the model rather than the results of the experiment itself. In fact the results are less interesting given that the data we used contain both healthy subjects and patients with different diseases.

The medial modeling is particular useful for population-wise comparison and analysis. Comparing with deterministic skeletonization, which yields discrete surfaces with uncertain number of vertices and uncertain number of branches that are sensitive to noises on the boundary, the medial model produces a robust approximation to the medial axis with consistent branching configuration and consistent surface representation. This simple and robust representation of the medial manifolds allows population-wise study and is also useful for visualization of statistical results. It is even possible to establish a shape-based coordinate system for each cardiac shape, which can be a much more detailed version of the standard sixteen segments of the left ventricle in polar plot (basal and middle areas: six segments, apical: four segments) that is a coarse division based on simple geometric measures.

Another important potential application of cardiac medial model is on learning based cardiac image segmentation. Active shape model (ASM) [1] is widely used for statistical shape and appearance modeling adopted in prior based segmentations. It has also been successfully applied on cardiac images [12. Comparing with ASM, medial model addresses the coupling of shape and appearance 
features better by sampling the appearance features in a shape based coordinate system [1]. The medial model also has the advantage to explicitly model the wall thickness, an important shape features for cardiac shapes. The application of medial model on cardiac segmentation is now under investigation.

\section{Acknowledgement}

Medial methodology development is funded by the NIH grants AG027785 and NS061111. We also acknowledge support from Spanish MEC (TEC2006-03617), ISCIII (FIS2004/40676), and CDTI(CENIT-CDTEAM); as well as Dr. Marina Huguet from CETIR - Sant Jordi Cardiovascular Centre (Barcelona, Spain) for kindly providing the cine MR data sets used in this work.

\section{References}

1. Yushkevich, P.A., Zhang, H., Gee, J.: Continuous medial representation for anatomical structures. IEEE Trans. Med. Imaging 25(2), 1547-1564 (2006)

2. Pizer, S.M., Fletcher, P.T., Joshi, S., Thall, A., Chen, J.Z., Fridman, Y., Fritsch, D.S., Gash, A.G., Glotzer, J.M., Jiroutek, M.R., Lu, C., Muller, K.E., Tracton, G., Yushkevich, P., Chaney, E.L.: Deformable m-reps for 3D medical image segmentation. International Journal of Computer Vision 55(2), 85-106 (2003)

3. Azhari, H., Sideman, S., Weiss, J., Shapiro, E., Weisfeldt, M., Graves, W., Rogers, W., Beyar, R.: Three-dimensional mapping of acute ischemic regions using MRI: wall thickening versus motion analysis. Am. J. Physiol. 259, H1492-1503 (1990)

4. Sun, H., Avants, B.B., Frangi, A.F., Ordas, S., Gee, J.C., Yushkevich, P.A.: Branching medial models for cardiac shape representation. In: Proceedings of IEEE International Symposium on Biomedical Imaging (2008)

5. Bolson, E., Sheehan, F.: Centersurface model for $3 \mathrm{~d}$ analysis of regional left ventricular function. In: Proceedings of Computers in Cardiology 1993, pp. 735-738 (September 1993)

6. Han, Q., Pizer, S.M., Merck, D., Joshi, S., Jeong, J.Y.: Multi-figure anatomical objects for shape statistics. In: Information Processing in Medical Imaging, pp. 701-712 (2005)

7. Terriberry, T.B.: Continuous Medial Models in Two-Sample Statistics of Shape. PhD thesis, University of North Carolina at Chapel Hill (2006)

8. Giblin, P., Kimia, B.: A formal classification of 3D medial axis points and their local geometry. In: IEEE Computer Society Conference on Computer Vision and Pattern Recognition, pp. 566-573 (2000)

9. Joshi, S., Davis, B., Jomier, M., Gerig, G.: Unbiased diffeomorphic atlas construction for computational anatomy. Neuro. image 23 (Suppl. 1), 151-160 (2004)

10. Avants, B.B., Gee, J.C.: Symmetric geodesic shape averaging and shape interpolation. In: ECCV Workshops CVAMIA and MMBIA, pp. 99-110 (2004)

11. Cootes, T.F., Taylor, C.J., Cooper, D.H., Graham, J.: Active shape models - Their training and application. Computer Vision. Graphics and Image Processing 61(1), 38-59 (1995)

12. Assen, H.C.V., Danilouchkine, M.G., Frangi, A.F., Ordas, S., Westenberg, J.J., Reiber, J.H., Lelieveldt, B.P.: Spasm: A 3D-ASM for segmentation of sparse and arbitrarily oriented cardiac MRI data. Medical Image Analysis 10(2), 286-303 